\title{
Rating or Sorting: terminology matters
}

\author{
A. Colorni $(*)$, A. Tsoukiàs $(+)^{1}$
}

(*) POLIEDRA, Politecnico di Milano

(+) LAMSADE-CNRS, PSL, Université Paris Dauphine

\begin{abstract}
In this paper we argue that the term "sorting" (in MCDA), although established within a well defined community, is unappropriate for linguistic, scientific and pragmatic reasons. We present and discuss such reasons and we suggest the use of the term "rating", since fitting better both the foundational part of this class of methods and the necessity to improve the visibility of our community.
\end{abstract}

\section{Introduction}

The expression "multiple criteria sorting" (or multi-criteria sorting) sounds familiar and seems clear to a number of scientists. Which ones? At least those publishing a paper in the present special issue, and probably also those reading it. To this community, we can also safely add most researchers that are loosely or closely related to the EURO MCDA Working Group ${ }^{2}$. This paper is aimed at this community and claims that the expression "multiple criteria sorting" (i) is not an accurate description of what we mean and (ii) is not effective for communicating with other communities that also perform some kind of multiple criteria sorting, but under another name.

\section{History and language}

In the early 70s, while Bernard Roy was developing his approach about decision aiding and the family of the ELECTRE methods, he started facing a number of decision problems (arising in real case studies) characterised by the presence of multiple criteria, but also by the presence of "predefined" categories to which the alternatives were expected to be assigned. A typical case being separating job

\footnotetext{
${ }^{1}$ Corresponding author: Alexis Tsoukiàs, LAMSADE-CNRS, PSL, Université Paris Dauphine, 75775 Paris Cedex 16, France; e-mail: tsoukias@lamsade.dauphine.fr

${ }^{2}$ https: / / www.euro-online.org/web/ewg/1/ewg-mcda-euro-working -group-on-multiple-criteria-decision-aiding
} 
candidates among the rejected, the accepted and the ones to be further discussed. A first result has been published (in French) in Moscarola and Roy (1977), concerning "trichotomic" procedures aiming at partitioning a set in three predefined categories as mentioned before. Shortly after, at the LAMSADE, under the supervision of Bernard Roy, Yu Wei wrote his PhD Thesis studying a new ELECTRE method, since then called Electre Tri (see Yu, 1992). We can consider this as a milestone, establishing a whole area of decision aiding methods using multiple criteria which has been named, essentially within the EURO MCDA Working Group, "Sorting Methods". So far for the origins.

It is interesting to note that most of the original work has been done in French, adopting the term "tri" (from the French verb "trier") which could be translated as "assigning objects to groups characterised by some properties". Interestingly enough, the term does not make any reference to the fact that such groups should be ordered. Indeed, in French, a typical use of the verb "trier" is in the sentence: "trier la correspondance" (to sort letters according to their destinations, typically streets or postcode areas). In any case, Bernard Roy, for reasons we do not know, decided that the English translation of this term would be "sorting" and with this term he completed what was (for him) the three possible "problématiques" (never translated by Bernard in English): choice, sorting and ranking.

Consulting the Cambridge Dictionary (Sort, 2020), we get for the word sorting: "to put a number of things in an order or to separate them into groups", with examples such as:

- Paper, plastic, and cans are sorted for recycling;

- I'm going to sort these old books into those to be kept and those to be thrown away;

- You can use the computer to sort the newspaper articles alphabetically, by date, or by subject;

- She found the ring while sorting (through) some clothes.

It is interesting to note that the term sorting does not necessarily imply that the "groups" within which the elements are "sorted" are ordered. They can be (e.g. articles sorted by date) but they need not (e.g. articles sorted by subject).

Concluding: an easy linguistic check shows that the two terms ("tri" in French and "sorting" in English) could be considered equivalent (actually, any automatic translator will find them equivalent). However, the meaning of these two terms does not correspond to the original problem for which these decision aiding methods have been designed: assigning objects to categories which exist independently 
of the objects and which are ordered between them. In other terms, we keep using these two terms (in the two languages), while they do not correspond to what we actually try to do.

\section{Problem Statements}

So far for the linguistics. Let us now move to a more formal discussion layer. One of the major contributions of Bernard Roy to Decision Aiding has been the intuition that there are several different types of "decision problems" beyond the classical idea that deciding means choosing the optimal, the best, or whatever unique solution. For this purpose he invented the term "problématique" (in French) and never gave a formal definition of this term (and never really attempted to translate it into English). He considered this term to be self evident, leaving a degree of ambiguity on how to interpret this topic. Hereafter, we will translate "problématique" as "problem statement" and we claim that, within a formally established methodology, such terms need to be clearly defined. Let us recall that in addition to the standard choice problem statement, Roy defined the ranking problem statement and the sorting problem statement (Roy, 1985).

Besides axiomatic reasons for using clearly defined terms, there are pragmatic and algorithmic ones: we need to know when, how and for which purpose we may use methods, protocols and algorithms which may fit a problem statement, but not another.

The notion of problem statement has already been introduced in Bouyssou, Marchant, Pirlot, Tsoukiàs, and Vincke (2006); Tsoukiàs (2007); Colorni and Tsoukiàs (2013). Any decision problem we may consider (both in theory and in practice) boils down to constructing a set $A$ and then partitioning it in $n$ classes. The concept of partition is well defined and considering (from a formal point of view) a decision as the partitioning of a set is simple and robust: it applies equally well in presence of fuzzy sets (fuzzy partitions) and in presence of different forms of uncertainty, including the case where an element of the set $A$ could belong to a union of several equivalence classes (of the set $A$ ) without knowing which one precisely.

There are several ways in which the partitioning of $A$ can be done, resulting in different ways to construct the equivalence classes $[A]_{i}(i=1, \ldots, n)$ partitioning $A$. We focus on two different types of choices.

1. The first choice concerns the "primitives" (minimal strictly necessary information) used in order to construct the classes of the partition. Such primi- 
tives come under the form of a binary relation denoted by $S$ and for which we have two options:

- $S$ is a relation defined on $A$, in other terms $S \subseteq A \times A$, and we call this a "relative comparison";

- $S$ is a relation comparing the elements of $A$ to some externally defined norms (or standards) and vice versa. More precisely, if $N$ is the set of norms, then $S \subseteq A \times N \cup N \times A$, and we call this an "absolute comparison".

2. The second choice concerns the existence or not of an "order" on the classes of the constructed partition. Let us make this point clearer. Consider $[A]=$ $\left\{[A]_{1}, \ldots,[A]_{n}\right\}$ the set of all classes partitioning $A$. Let us define a binary relation $\succcurlyeq \subseteq[A] \times[A]$ of the type "at least as good as", separable in a symmetric part $(\sim)$ and an asymmetric $(\succ)$ part. We have two cases:

- $\succcurlyeq=\emptyset$ which amounts saying there is no order among the classes;

- $\succcurlyeq$ is reflexive, antisymmetric and transitive (a partial order), with a nonempty asymmetric part $\succ$. In this case, we consider the classes as (at least partially) ordered. The reader will note that this option is compatible with stronger ordered structures such as complete orders or even complete orders admitting a numerical representation on an interval scale or ratio scale. However, such strong ordered structures are not necessary.

Combining the above two choices, we can define four basic problem statements:

- ranking (partitioning in ordered classes, using relative comparisons);

- rating (partitioning in ordered classes, using absolute comparisons);

- clustering (partitioning in unordered classes, using relative comparisons);

- assignment (partitioning in unordered classes, using absolute comparisons).

For all the above cases, there are at least two special subcases:

- when the classes are just two (complementary);

- when one or more classes have a given cardinality. 
For instance, a "choice" problem statement is a ranking problem statement with only two classes: the elements to be chosen and the rest. The reader will note that most optimisation problems fit the ranking problem statement with only two classes (the chosen/optimal ones and the rest). Equally easy is to realise that most pattern recognition, diagnosis signal processing, and classification problems fit the assignment problem statement, while most data analysis problems correspond to either clustering or rating problem statements.

Turning back to our linguistic distinctions, it is interesting to note that what Bernard Roy used to call "sorting" corresponds to the definition of rating, while the term sorting perhaps better fits the assignment problem statement. The advantage of using a formal definition of problem statements is that we can now clearly state what type of decision problem we are handling and check whether the methods, protocols, algorithms we aim at using, fit the requirements of such problem statements.

\section{Pragmatic considerations}

Our claim is that we should stop using the term sorting and adopt the term rating, despite the "history" of the term sorting. Our first argument is that the term rating has a precise theoretical foundation and corresponds exactly to the problem for which all the "sorting" methods in the MCDA literature have been designed. We understand that there might be counterarguments about our theoretical framework, but these we leave them for a more thorough theoretical discussion (welcome in any case). However, there might be a reasoning of the type: "after all the term sorting is now established, why should we take care to change it?" It is this counterargument we want to discuss in the following.

It is true that the term sorting is now established. But where? Within a small (although prestigious) community which is the EURO MCDA Working Group proudly established by Bernard Roy more than 45 years ago. The fact is that the rest of the world has rating problems, uses rating procedures and talks about rating. Just consider the world of finance: people talk about credit rating, debt rating, risk rating, countries or companies rating. Consider the business of recommender systems: people talk about product rating, vendor rating, platforms rating, systems rating etc. Consider topics such as environmental assessment and public policies where people rate vulnerability, resilience, impacts etc.

In almost all these case, rating can be seen as a process of ordinal measurement, that is the mapping of the elements of $A$ to a set of totally ordered labels 
(the grades of the ordinal scale). This process yields classes which are also totally ordered. Conversely, ordinal measurement can be considered as a rating problem. However, rating can be less than ordinal measurement (in case only a partial order is established among the classes) or more than ordinal measurement (in case we can measure the distance among the classes as e.g. with measurable multiattribute value functions (Dyer \& Sarin, 1979)).

Interestingly enough, the term rating has already been adopted in (von Winterfeldt \& Edwards, 1986) and in (Keeney \& von Winterfeldt, 2007) when procedures such as "simple rating" are adopted, while the terms grading and scoring are also adopted in some practitioners areas (such as credit scoring). Any of such terms lacks however, a formal definition.

Our argument is thus simple. We can keep calling ourselves experts of sorting methods and keep being ignored by the communities interested by rating, or we can change our label in rating (which is what we do) and become appropriately visible.

\section{Conclusion}

Bernard Roy left a huge legacy on theoretical as well as practical topics to work with. The problem of assigning objects/alternatives/options to predefined and ordered categories is among the big avenues opened within this legacy and this has been followed by dozens of estimated colleagues. It is time however, to realise that in order to pursue further this legacy and keep innovating in our theory and practice, we also need to connect to what other communities do in our neighbourhood. And for this purpose we need to be precise about our qualifications: we develop rating methods. Terminology matters.

\section{Acknowledgement}

This essay benefitted from several discussions with many friends and colleagues, the list being too long to recall here. The guest editors provided very helpful comments which improved very much the text and clarified our purposes and we desire to acknowledge their help. 


\section{References}

Bouyssou, D., Marchant, T., Pirlot, M., Tsoukiàs, A., \& Vincke, P. (2006). Evaluation and decision models with multiple criteria: Stepping stones for the analyst. Boston: Springer Verlag.

Colorni, A., \& Tsoukiàs, A. (2013). What is a decision problem? preliminary statements. In Proceedings of ADT 2013, LNAI 8176 (p. 139 - 153). Springer Verlag, Berlin.

Dyer, J.S., \& Sarin, R.K. (1979). Measurable multiattribute value functions. Operations Research, 27(4), 810-822.

Keeney, R., \& von Winterfeldt, D. (2007). Practical value models. In W. Edwards, R. Miles, \& D. von Winterfeldt (Eds.), Advances in decision analysis: From foundations to applications (p. 232 - 252). Cambridge, UK: Cambridge University Press.

Krantz, D.H., Luce, R.D., Suppes, P., \& Tversky, A. (1971). Foundations of Measurement. Additive and polynomial representations. New York and London: Academic Press.

Moscarola, J., \& Roy, B. (1977). Procédure automatique d'examen de dossiers fondée sur une segmentation trichotomique en présence de critères multiples. RAIRO Recherche Opérationnelle, 11(2), 145-173.

Roy, B. (1985). Méthodologie multicritère d'aide à la décision. Paris: Economica.

Sorting. (2020). Cambridge dictionary. Cambridge University Press, Cambridge.

Tsoukiàs, A. (2007). On the concept of decision aiding process. Annals of Operations Research, 154, 3 - 27.

von Winterfeldt, D., \& Edwards, W. (1986). Decision analysis and behavorial research. Cambridge: Cambridge University Press.

Yu, W. (1992). Aide multicritère à la décision dans le cadre de la problématique du tri: méthodes et applications (Unpublished doctoral dissertation). LAMSADE, Université Paris Dauphine, Paris. 